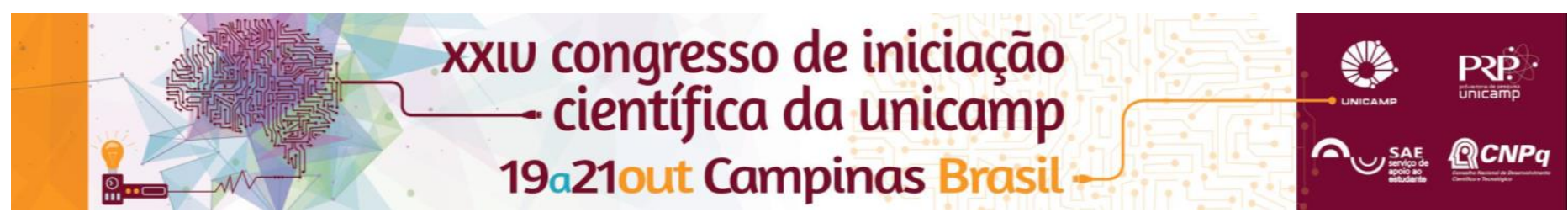

\title{
Hydrogen-1 Nuclear Magnetic Resonance Spectroscopy applied to metabolomics of the crack users and patientis with schizophrenia
}

\author{
Rafael N. de Souza*, João G. M. Pontes, Ljubica Tasic.
}

\begin{abstract}
This research aims to compare metabolomic profiles of blood serum samples of healthy people $(\mathrm{HC})$ to those who are addicted to crack and seconderaly, metabolomic profiles of blood serum samples of patientis with schizophrenia (SCZ) to those addicted to crack using nuclear magnetic resonance spectroscopy ( ${ }^{1} \mathrm{H}$ NMR). ${ }^{1} \mathrm{H}$ NMR data shall be then treated using chemometric tools such as principal component analysis (PCA) aiming to differentiate two groups of samples and enable identification of NMR spectral regions important for the metabolic differences. Further, biomarkers for crack dependence might be identified and, thus, improve clinical diagnosis and discovery of new pharmacological and therapeutic interventions in this drug dependence treatment. A differential diagnosis may be used to differentiate the symptoms caused by the crack dependence to the positive symptoms of schizophrenia patients, such as psychosis.
\end{abstract}

\section{Key words:}

Nuclear Magnetic Resonance, Metabolomics, Crack users.

\section{Introduction}

Crack is a different administration and presentation of cocaine. It is a strong stimulant of the sympathetic nervous system and has a capacity to cause addiction in human beings due to it's quick interaction with central nervous system. Since 1990's, Brazil faces the increase in the number of crack users ${ }^{1}$ and the serious public health problem caused by the lack of treatment of these users.

Therefore, this study aims to compare the metabolomics profiles of $\mathrm{HC}$ with crack users in order to find potential biomarkers for this dependence.

It is also known that some drugs, such as crack, mimic some characteristic symptoms of mental illnesses such as hallucinations in schizophrenia ${ }^{2}$. By comparing the metabolomic profile of crack users with SCZ we intend, in a pioneer research, to find possible metabolites that differentiate the symptoms generated by the crack dependence and symptoms of schizophrenia.

\section{Results and Discussion}

The blood serum samples were collected and processed by our collaborators from the Department of Psychiatry from Federal University of São Paulo (UNIFESP) and stored in biofreezer at $-80^{\circ} \mathrm{C}$ for not more than 10 days before NMR analyses were performed.

Twenty-six (26) blood serum samples of HC and SCZ were analyzed and also eleven (11) blood serum samples from the crack users. The ${ }^{1} \mathrm{H}$ NMR analyses were made in triplicate using deuterium oxide $\left(\mathrm{D}_{2} \mathrm{O}\right)$ as a solvent in blood serum sample preparation and recorded in a Bruker AVANCE III (600 MHz) spectrometer at $25^{\circ} \mathrm{C}$ using the TBI probe.

Analysis of ${ }^{1} \mathrm{H}$ NMR spectra of the three groups indicates that there are regions with some differences, as in 2.00 to $4.00 \mathrm{ppm}$. This region enabled that in more refined analyses by PCA and PLS-DA, potentials biomarkers could be pointed.

Two PCA were performed with $\mathrm{HC}$ and group of crack users and SCZ and crack users. There was a significant separation between three groups. Then, an analysis was made using Heteronuclear Single Quantum Coherence (HSQC) NMR data and several correlations were found and then was initiated the search for possible biomarkers for crack dependence.

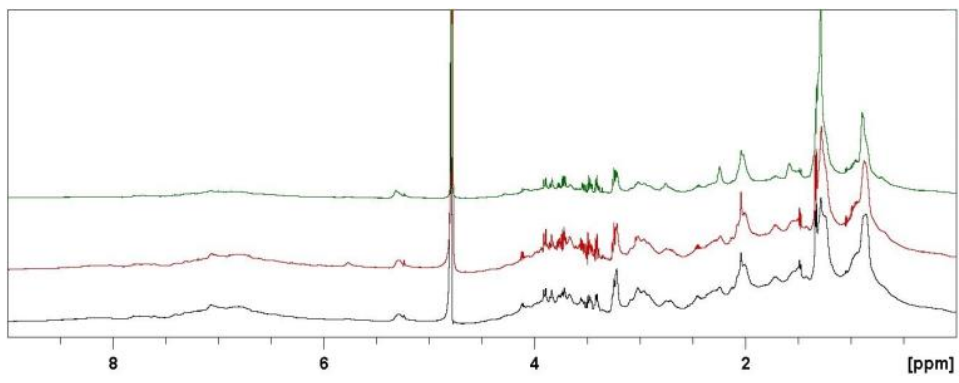

Figure 1. Examples of the ${ }^{1} \mathrm{H}$ NMR spectra (ns $=128$ ) of blood serum samples: control healthy group (black), crack user (red) and patient with schizophrenia (green).
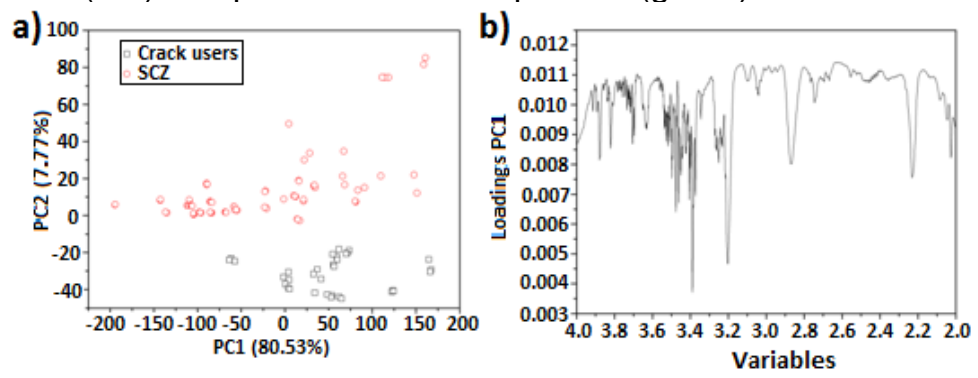

Figure 2: PCA a) Graph scores - crack users (black) and patients with schizophrenia (red); b) Graph Loadings - $\delta$ 2.00 to 4.00 .

\section{Conclusions}

The NMR specral region which provoked greater separation in chemometric analysis between $\mathrm{HC}$ and crack users, and between SCZ and crack users was the region between 2.00 and $4.00 \mathrm{ppm}$. The results of HSQC of $\mathrm{HC}$ and crack users are being used in a search for biomarkers using Biological NMR Data Banks.

\section{Acknowledgements}

CNPq: 454234/2014-7 / FAPESP: 2014/18938-8 Parecer consubstanciado do CEP: 842.072

${ }^{1}$ Schneider, J.; Lisboa, C.; Mallmann, C. Psiquiatria 2014, 3, 24-33. 2 Costa, S. N; Machado, S. M. D.; Rev Med. Minas Gerais 2012; 22(2): 199-205. 